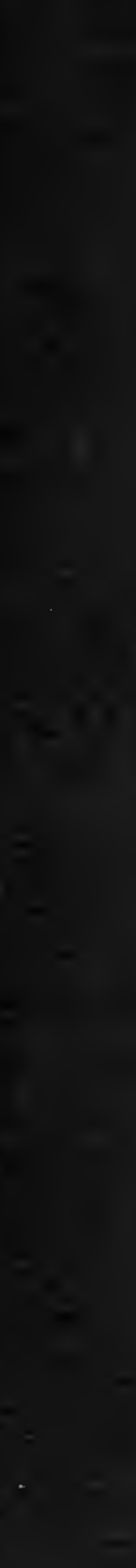


Digitized by the Internet Archive in 2007 with funding from Microsoft Corporation 
UNIVERSITY OF CALIFORNIA PUBLICATIONS

IN

AMERICAN ARCHAEOLOGY AND ETHNOLOGY

Vol. 14, No. 5, pp. 489-502

May 31, 1919

\section{THE GENETIC RELATIONSHIP OF THE NORTH AMERICAN INDIAN LANGUAGES}

BY

PAUL RADIN 


\section{UNIVERSITY OF CALIFORNIA PUBLICATIONS DEPARTMENT OF ANTHROPOLOGY}

The following publications dealing with archaeological and ethnological subjects issued under the direction of the Department of Anthropology are sent in exchange for the publications of anthropological departments and museums, and for journals devoted to general anthropology or to archaeology and ethnology. They are for sale at the prices stated. Fxchanges should be directed to The Exchange Department, University Iibrary, Berkeley, California, U.S. A. All orders and remittances should be addressed to the University of California Press.

AMERICAN ARCHAEOTOGY AND ETHNOLOGY.-A. I. Kroeber, Editor. Prices, Volume 1, \$4.25; Volumes 2 to 11, inclusive, \$3.50 each; Volume 12 and following, $\$ 5.00$ each.

Cited as Univ. Calif. Publ. Am. Arch. Ethn.

Price

Vol. 1. 1. Life and Culture of the Hupa, by Pling Earle Goddard. Pp. 1-88; plates 1-30. September, 1903

2. Hupa Texts, by Pliny Earle Goddard. Pp. 89-368. March, $1904 \ldots . . . . . . . . . . . . . . \quad 3.00$ Index, pp. 369-378.

Vol. 2. 1. The Exploration of the Potter Creek Cave, by William J. Sinclair. Pp. 1-27; plates 1-14. April, 1904

2. The Languages of the Coast of California South of San Francisco, by A. I. Kroeber. Pp. 29-80, with a map. June, 1904

3. Types of Indian Culture in Califormia, by A. I. Kroeber. Pp. 81-103. June, 1904

4. Basket Designs of the Indians of Northwestern California, by A. Kroeber. Pp. 105-164; plates 15-21. January, 1905.

5. The Yokuts Ianguage of South Central Califormia, by A. I. Kroeber. Pp. 165-377. January, 1907

Index, pp. 379-392.

Vol. 3. The Morphology of the Hupa Ianguage, by Pliny Earle Goddard. 344 pp. June, 1905

Vol. 4. 1. The Earliest Historical Relations between Mexico and Japan, from original documents preserved in Spain and Japan, by Zella Nuttall. Pp. 1-47. April, 1906

2. Contribution to the Physical Anthropology of California, based on collections in the Department of Anthropology of the University of Califormia, and in the U. S. National Museum, by Ales Hrdlicka., Pp. 49-64, with 5 tables; plates 1-10, and map. June, 1906

3. The Shoshonean Dialects of California, by A. I. Kroeber. Pp. 65-166. Eebruary, 1907

4. Indian Myths from South Central California, by A. I. Kroeber. Pp. 167250. May, 1907

5. The Washo Ianguage of East Central California and Nevada, by A. I. Kroeber. Pp. 251-318. September, 1907

6. The Religion of the Indians of California, by A. I. Kroeber. Pp. 319-356. September, 1907 .............. Index, pp. 357-374.

Vol. 5. 1. The Phonology of the Hupa Language; Part I, The Individual Sounds, by Pliny Earle Goddard. Pp. 1-20, plates 1-8. March, 1907

2. Navaho Myths, Prayers and Songs, with Texts and Translations, by Washington Matthews, edited by Pliny Earle Goddard. 'Pp. 21-63. September, 1907

3. Kato Texts, by Pliny Earle Goddard. Pp. 65-238, plate 9. December, 1909

4. The Material Culture of the Klamath Lake and Modoc Indians of Northeastern California and Southern Oregon, by S. A. Barrett. Pp. 239-292, plates 10-25. June, 1910

5. The Chimariko Indians and Ianguage, by Roland B. Dixon. Pp. 293-380. August, 1910 Index, pp. 381-384.

Vol.6. 1. The Ethno-Geography of the Pomo and Neighboring Indians, by Samuel Alfred Barrett. Pp. 1-332, maps 1-2. February, 1908 ......................

2. The Geography and Dialects of the Miwok Indians, by Samuel Alfred Barrett. - Pp. 333-368, map 3.

3. On the Evidence of the Occupation of Certain Regions by the Miwok Indians, by A. I. Kroeber. Pp. 369-380.

Nos. 2 and 3 in one cover. February, 1908 Index, pp. $381-400$. 


\section{UNIVERSITY OF CALIFORNIA PUBLICATIONS}

in

AMERICAN ARCHAEOLOGY AND ETHNOLOGY

Vol. 14, No. 5, pp. 489-502

May 31,1919

\section{THE GENETIC RELATIONSHIP OF THE NORTH AMERICAN INDIAN LANGUAGES ${ }^{1}$}

$B Y$

PAUL RADIN

All studies of the Indian languages of North Ameriea go baek properly to J. W. Powell's paper in the Seventh Annual Report of the Burcau of American Ethnology. Unfortunately his preliminary study was interpreted by most Amerieanists as demonstrating that genetic relationships between the stoeks he postulated had been disproved, or at least were to be presumed as disproved, whereas all he in reality elaimed was that the relationship had not been determined. This attitude toward Powell's elassifieation was strengthened by the results of Boas's investigations. The task Professor Boas and his sehool set themselves was the analytical presentation of the various Indian languages aecording to the speeifie genius of each, and interest thus shifted entirely from what were deemed futile genetie problems to monographie studies of special languages.

As might have been anticipated, however, hints and suggestions as to possible relationships were made many years ago. The grouping of Nahuatl with Shonshonean, first diseussed by Busehmann, was pressed by Brinton, and the latter likewise first postulated the relationship of Yuman and Tequistlateean (Chontal). Boas himself pointed ont resemblanees between Athapaskan, Haida, and Tlingit, for whieh genetic unity was subsequently proved by Sapir. He also showed definite struetural similarities between Kwakiutl, Salish, and Chemakum. The first serious reduetion of the number of stoeks was, however, that proposed by Kroeber and Dixon, who, subsequently

1 This paper is arowedly preliminary and no attempt has been marle to consistently follow all the morphological forms and the vocabularies through all the languages. Owing to space limitations much has had to be omitted; in particular the proof for the existence of those morphological elements isolated by myself, which had previously not been recognized as such. 
aided by Sapir and Harrington, reduced all the languages of California to seven stocks. One of the most startling and brilliant achievements in this connection was Sapir's demonstration of the relationship of Wiyot and Yurok to Algonkin. Harrington subsequently connected Tanoan, Kiowan, and Shoshonean. Thell followed Swanton's suggestion as to a possible relationship between Siouan and MuskhogeanNatchez having been previously included by him in the latter-and his demonstration of the relation of Atakapa, Tonkawa, and Coahuiltecan, a group that Sapir subsequently, in an unpublished paper, has shown fairly definitely to be connected with Hokan. The latter author has likewise arrived at the conclusion that Takelma, Coos, Chinook, and Tsimshian belong to the Penutian group. Other vaguely suggested relationships were those of Kutenai with Algonkin, Iroquois with Caddoan, and Yuki with Penutian (Kroeber).

If all these groupings were to be accepted the linguistic stocks in North America north of the Rio Grande would be practically reduced to Athapaskan, Hokan, Penutian, Kwakiutl-Salish, Kutenai-Algonkin, Shoshonean-Tanoan, Iroquoian-Caddoan, Siouan-Muskhogean, Lutuamian, Sahaptin, Zunian, and Keresan. The postulation of the genetic relationship of all the languages of North America contained in the following paper will then, considering these previous tentative conclusions, hardly scem so revolutionary.

The present paper is the result of the writer's detailed study of Wappo, a Yukian language, which showed rather clear and definite relationships to Hokan, on the one hand, and to Siouan, on the other, and subsequently disclosed remarkable similarities to Athapaskan and Penutian. A comparison of Winnebago (Siouan) with the unpublished grammar of Southern Paiute (Shoshoneon), ${ }^{2}$ afterwards undertaken, showed marked resemblances between Siouan and Shoshonean.

The morphological elements and vocabularies that follow speak for themselves. The resemblances seem clearly of a fundamental nature, including, for instance, the impcrative, passive, continuative, causative, dubitative, nominal sign, formation of plural, formation of tenses, the reflexive, interrogative, the demonstratives, the personal pronouns, and many of the common nouns and verbs. There are but two possible explanations : borrowing or genetic relationship. Borrowing is practically excluded, particularly in the case of the morphological elements, because of the universal mamner of their occurrence

2 Kindly placed at the writer's disposal by Dr. Sapir. 
all over North Ameriea, and it is equally impossible to attribute to chance so many startling rescmblanees not only of meaning but even of position in the word unit.

One feature in particular I wish to point out, namely, the marked prominence of verb "aspeets." These had, of course, been recognized before. To me they seem to be among the most fundamental characteristies of the languages of Nortl America. There appear to be at least six: the momentaneous, durative-usitative, transitive, intransitive (often medio-passive), causative, and continuative, the last five being frequently associated with the suffixes $\mathrm{s}$ or $\mathrm{e} ; \mathrm{t} ; \mathrm{k} ; \mathrm{n}$ or $\mathrm{t}$; and $\mathrm{l}$. Frequently these elements are definitely suffixed to certain verbs. They have been so far determined for Athapaskan, Hokan, Yukian, Penutian, Siouan, and Kwakiutl.

The general conclusion advanced in this paper is corroborated likewise when the linguistic stocks are grouped according to specific traits. Let us for instanee select the following cight fundamental traits: (1) objeetive case ending; (2) subjective and objective personal pronouns; (3) different stems for singular and plural of verbs; (4) instrumental prefixes; (5) passive voice; (6) irregular formation of plural; (7) reduplication; (8) suffixes or infixes expressing verb aspcet.

1. Penutian, Shoshonean, Yukian, Siouan, Algonkin, Kwakiutl, Sahaptin, Lutuamian, Muskhogean.

2. Yukian, Hokan, Atlapaskan, Siouan, Muskhogean, Iroquian.

3. Athapaskan, Hokan, Yukian, Shoshonean, Siouan (Biloxi), Muskhogean, Tsimshian, Chinookan, Salish.

4. Athapaskan, Shoshonean, Hokan, some Penutian languages, Hokan, Siouan, Musklogean, Yukian, Iroquoian.

5. All.

6. Practically all.

7. All.

8. All.

A cursory glanee at the above groupings shows that at best the languages fall into two groups, one having an apparent differentiation of the personal pronouns into a subjeetive and objective series. It might be well to point out, however, that all those languages whieh seem to have but one personal pronominal series have a series of suffixed pronominal enclities, whieh often diselose forms for the first and second singular quite distinet from the independent personal pronouns, and these latter have frequently the function of subjeetive personal pronouns.

Any attempt at defining very clearly the more intimate groupings 
of these languages is bound to be premature; but I would like to suggest three sub-groups.

I. Salish, Kwakiutl, Kutenai, Algonkin.

II. Penutian (Sapir's enlarged group), Lutuamian, ${ }^{3}$ Sahaptin, Shoshonean, Tanoan, Yukian, Mixe, ${ }^{4}$ Zapotecan, Caddoan, Iroquoian.

III. Athapaskan, Hokan, Maya, ${ }^{5}$ Siouan, Muskhogean.

Athapaskan will probably turn out to be equally distant from Hokan, on the one hand, and Tsimshian and Salish, on the other; Yukian equally distant from Athapaskan, Hokan, and Siouan, but closest to Penutian; Shoshonean closest to Tanoan and Siouan, but equally distant from both Penutian and Yukian; and Iroquoian closest to Algonkin, but equally distant from Caddoan and Muskhogean.

Finally it may be argued that general ethnological considerations corroborate the conclusion of a genetic relationship of the languages in question. Most recent American archaeologists seem to be agreed that 15,000 years is an adequate maximum for the settlement of North America by the Indians. That implies very definitely that the assumed differentiation into fifty-eight distinct stocks must have taken place in northeastern Asia, the assumed home of our aborigines. However, there is no evidence either in the present or past linguistic picture of northeastern Asia that would justify us in assuming a large number of distinct languages, and we are consequently forced to the conclusion that the differentiation in North America is secondary and took place after the settlement of the continent. ${ }^{6.7}$

3 This and Sahaptin belong elearly to Sapir's enlarged Penutian group.

4 This and Zapotecan belong fairly definitely to Uto-Aztecan.

5 Belongs fairly definitely with Hokan.

${ }^{6}$ One word about the morphological elements presented. Except where indicated no morphological elements are given that have not been recognized as such by the authorities from whom they are quoted. (R) indicates manuseript data in my possession, and (S) indicates Sapir. In general, where a particular language belonging to a large group is given the form selected has been one that seemed typical.

7 The following abbreviations are used. The forms are taken from the standard authorities.

Al., Algonkin; Ojib., Ojibwa; Wiy., Wiyot; Yur., Yurok.

Ath., Athapaskan; Hu., Hupa; Ka., Kato; Be., Beaver; Mon., Montagnais.

Cad., Caddoan.

Hai., Haida.

H., Hokan; Chim., Chimariko; Ka., Karok; Po., Pomo; Sa., Salinan; Was., Washo; Ya., Yana.

Iro., Iroquoian.

Kut., Kutenai.

Knaw., Kwakiutl.

Lut., Lutuamian; Kla., Klamath.

M., Muskhogean; Choc., Choctaw.

P., Penutian; Cost., Costanoan; Mai., Maidu; Miw., Miwok; Mut., Mutsun; 
In conclusion it may be stated that the data adduced in the present paper, elearly demonstrate, in the author's opinion, the genetic relationship of all Indian languages of North America.

\section{MORPHOLOGICAL ELEMENTS}

1. Agentive; -pe, P.; -pan, P. Mut.; pï, Pai.; -pi, Si. Dak.

2. Agentive; -ił, Siusl.; -le, Y. Wap.; -xale, H. Po.; -al, Maya.

3. At, in; -mpa, Pai.; -pa, Lut. Kla.; -bai, H. Po.; -op, Y. Yu.; -pa, Chin.; -pai, P. Mai.; me-, Ath. Hu.; pa, pan, Maya; pin, Al. Ojib.

4. Causative; -eg‘a, H. Po.; qa, Kwak.; -ke, Si. Win.; ka-, Tling.

5. Causative; -ta, Y. Wap.; -ta, P. Yok.; -t-, H. Sal. ; -et, Co. ; it, (to make) Kut.; -tui, Pai.; -ra, S. Win.; -n-, Ath. Ka.; -n-, Tsim.; -n-, Tak.; -atho, Wiy.; -t-, Al. Fox.

6. Causative; -si, Y. Wap.; -se, P. Mut.; -se, Tsim.

7. Comitative; -ka, Y. Wap.; -kö, P. Miw.; -kan, P. Mai.; -ka, Ath. Hu.

8. Continuative; -ad, P. Yok. (other dialects have -al); -ała, Kwak.; -l, Tsim.; -1, Tak.; -L, Chin.; -1, Ath. Hu.; -l, Y. Wap.; -1, Si. Dak. (with demonstratives); -l, H. Po. (R); -t, M. Choc.; do-, Wiy.; ru-, Cad.

9. Diminutive; -ite or tei, found in almost all languages.

10. Dubitative; -kuna, Siusl.; -guni, Si. Win.; -kane, P. Mut. (conditional); -k'i', Tak.; -g.ê, Tsim.; -qa, Kwak. (conditional); ru, Pai. (irrealis); -kwen, Y. Wap.; -k-, Al. Fox, (sign of potential); goni-ma, Al. Ojib.

11. Dubitative; -pi, H. Po.; -mpi, Pai.; amī, Tsim.; -ma, Y. Wap.

12. Durative; -is, Siusl.; -s-, P. Mut.; -x, Chin. (eustomary); -c, Ath. Ka.; -s-, Y. Wap.; s'a, Si. Win.; z-, Iro.

13. Durative, -mi, Pai.; '-mi, P. Miw.; -ki-ma, H. Po.; -mi, Y. Wap.

14. Easily; a-, Tsim.; -a, Pai.

15. Entirely; cu-, Tling.; eu, Pai. (very).

16. Fire (as prefix); d-, Ath. Hu.; da-, Si. Win.

17. For; -ki, H. Po.; -nqi, Pai.; -ki, Si. Win.; - ra (Nadene).

18. Frequentative; -ta, P. Yok.; -it, Sinsl.; -tau, Ath. Hu.; ta, Wiy.; -te, Iro.

19. From; -umpa, H. Sal.; -pa, Chin.; -bai, Tak.; -bue, uwe, H. Was.; -awa, H. Po.

20. Future; -bae, H. Po.; -mpa, Pai; -ma, P. Mai.; -pa, Y. Yu; op. Huave, -wi, wa, Al. Wiy.

21. Future; to, H. Po.; -tūx, Siusl.; -tsE-n, tE-n, Sa.; dE-m, Tsim.; -L, Kwak.; -ta, P. Mai.; -te, Ath. Hu.; ts-, Kut.; -t'e, Si. Po.; tei, M. Choc.; -ust, Cad.

22. Future; -si, Y. Wap.; -ee, H. Ka.; -aca, H. Was.; hi, M. Choc.; hi, P.; -s, Nah.; s-, Zap.

23. gu-, Tling.; -ke, Si. Dak. -ke, H. Po. (R); ki-, Yur.; k’a, Maya; ke-, Zap.; ga-d, Al. Ojib.; -ka, Ath. Be.

24. Hortatory; -pe, P.; -mE, Siusl.; ōp, Tsim.

25. Imperative; -ye, H. Was.; -i, P. Mut. ; -ย, Y. Wap. ; -yo, Si. Dak.; -ya, Pai.; $-\mathrm{i}$, Wiy.

Win., Wintun; Yok., Yokuts; Co., Coos; Chin., Chinook; Tak., Takelına; Tsim., Tsimshian.

Sal., Salish.

S., Shoshonean; Pai., Southern Paiute; Nah., Nahuatl.

Si., Siouan; Bil., Biloxi; Dak., Dakota; Pon., Ponka; Win., Winnebago.

Siusl., Siuslawan.

Tling., Tlingit.

Y., Yuki; Wap., Wappo; Yu., Yuki.

Zap., Zapotec. 
26. Imperative, -ił, Siusl.; -la, Kwak.; -la, Y. Wap.

27. Imperative; m-, H. Sal.; -mi, H. Po.; -mє, Y. Wap. ; -p, P. Mai.; -we, P. Mut.; -ba, Tak.; -m, Kut.; -po, Si. Dak.; en, Maya (em in other dialects).

28. Imperative; -t, P. Mut.; -ti, Y. Wap.; -de, -re, Si. Win.; -tla, Sa.; -ne, Ath. Hu.; -de, Tling.; ta, Al. Ojib.; tsa-, Wiy.

29. Imperative; ka-, H. Was.; -'ki, Tak.; -gª, Kwak. ; -kil, Kut.; aqª, Pai.; ga, Si. Pon.; ka-, Wiy.; ik, M. Choc.; okan, Al. Obij.; ke-, Cad.

30. In; -lö, Tsim.; -l, H. Po.; -li, Nall.

31. In; -ye, Ath. (Nadene ge) ; -gi, Si. Win.; -ko, Nah.; -ak, H. Ka.; kut, Cad.; -ge, Iro.

32. Indefinite object; ho-, Y. Wap.; ho-, H. Po. (R); lıo-, Si. Win.; -o-, (?) Ath. Ka.; o-, Al. Ojib.

33. Indefinite object; na-, Y. Wap.; ma-, H. Po. (R); wa-, Si. Win. (other dialects have ma-); me-, Nadene (S).

34. Indefinite tense; -n, P. Mut.; -in, P. Yok.; -n, P. Miw.; -n, Tsim.; ni-, Chin.; ni, Kut.; -nan, Si. Win.; na-, Wiy.; -du, H. Po. (R); -ta, H. Sal.; -ti, H. Ka.; -ta, Y. Wap. (indefinite past) ; -tï, Pai.(?).

35. Instrumental; -ma, Pai.; -ma, Y. Wap.;-imak, H. Po.;-muk, H. Was.; iba, M. Choc.

36. Interrogative; ha, Y. Wap.; he, Si. Win.; hi, P. Miw.; -a, Tsim.; ho, M. Cloc.; ia, Wiy.; -a, Kwak.; ye-, Atl. Be.; ä-, (?) Al. Fox.

37. Interrogative; ta, Y. Wap.; tce. H. Po.; -ce, Si. Win. (other dialects have tee) ; -ti, P. Miw.; da, Nadene (S); ta, M. Choc.

38. Intransitive; k-, H.'Sal.; -ki, Y. Wap.; -ki, Chin. (Cf. 52.)

39. Iterative; ona, Y. Wap. (again); na-, Ath. Hu.

40. Negation; -ił, Siusl.; la, Y. Wap.; ła, Kwak.; ał, Tsim.; ła, Ath. Hu.; la, M. Choe.; la, Zap.

41. Negation; -ke, Si. Win. (forms in $\mathrm{k}$ are practically universal among all tribes not using 1 ).

42. Nominal suffix; -s, P.; -s, Y. Wap.; -s, Co.; -'s, Tak.; -c, -s, Ath. Be.;8 -tsi, Pai.; -tca, ${ }^{9}$ Si. Dak.; -s, Wiy.; -sh, Lut. Kla.

43. Nominal suffix; -ume, Co.; -ā'mū, Em, Siusl.; -m, Tak.; -ı, Maya; am-, Tsim. ; -Em, Kwak.; -ma, P. Mai.; -am, Kut.; -ma, Y. „Wap.; -pi, -pa, Si. Dak.; -vi, mpi, Pai.; -wi, H. Ya.; -ma, Al. Ojib.

44. Nominal suffix; -āu, Siusl.; -iye, Co.; -a, Y. Wap.; -i, H. Sal.; -a, Tak.; -i, P. Yok.

45. Nominal suffix or prefix; -k, k-, Ath.; P.; H.; Al.; Kwak.; Sa.; Kut., Iro.

46. Nominal suffix; -l, Tak.; -I, Ath. Be.;11 -l, Y. Wap.; -I, H. (S) ; -L, Wiy.; -ya, Si. Dak. (other dialects have -ra, -la); -1, P. Mut.;12 -sla, Iro.

47. Nominal suffix or prefix; -uni, Siusl.; -ni, H. Sal.; -an, H. Ka.; -n, P. Mut.; ne (nominal particle), Iro.; -în, Co.; -n, Tak.; An-, Ath. Be.; -n, -ni, Si. Dak.;13 -n, Maya; -an, -win, Al. Ojib.; -in, Wiy.; n-, Mixe; n-, Zap.

48. Objective case ending; -a, P. Yok.; -i, P. Miw.; -e, P. Cost.; -a, Y. Yu.; -a, Pai.; -yan, Si. Bil.; -a, M. Choc.

49. Off ; tsa-, H. (S) ; te'a-, Y. Wap.; tee-, Ath. Hu.; sa-, Tsim.

\footnotetext{
8 Isolated by myself.

9 Isolated by myself.

10 Isolated by myself.

11 Isolated by myself.

12 Isolated by myself.

13 Isolated by myself.
} 
50. On; -ki, H. Po.; -ak, H. Ka.; -k, Y. Yu.; -k, Clin.; -akw, Wiy.; -ik, Yur.; -kut, -ka, Atl. Hu.; akan, Si. Dak.

51. Passive; -ül-tx,14 Siusl.; -1, Kwak.; -1-, Kut.; -l, Atl. Hu.; -l, Y. Wap.; -1-, Sa.; -1-, M. Choc, -1-, Zap.; -l-, Nah.; -t'ï, Pai.; -it, P. Yok.; -etu, H. Po. (R) ; -tä- (middle voice), Al. Fox; -t-, Iro.

52. Passive; -k, H. Sal.; -k (passive partieiple); H. Po.; -k, Y. Wap.; -k'w (passive partieiple), Tak.; -ku, Kwak.; -qa, Pai.; gu, Al. Fox.

53. Passive; -p, P. Miw.; P. Cost.; -ime, Siusl.; -ap, H. Sal. (passive reflexive); iyem, Co.; -ma', Tak.; -mє, Y. Wap.; -pi, Pai. (passive partieiple); -pi, Si. Dak. (passive partieiple).

54. Past tense; -k, P. Miw.; P. Cost.; -yak, P. Mai.; -kun, P. Mut.; ga-, Chin.; -ke, H. Po.; -qa, Pai.; -ki, Si. Win.; x-, Maya; k-, Zap.; gi-, Al. Ojib.; kits, Yur.; uks, Cad.

55. Past tense; o-, Tling.; o-, Y. Wap.; o-, Ath. Hu.; o-, Mixe; o-, Nah.

56. Past tense; -ta, P. Miw.; P. Cost.; -to, H. Sal.; -at, H. Ka.; -ta, Y. Wap.; -t'a', Tak.; -etl, Sa.; Lā, Tsim.; -uł, Kwak.; -t, Chin.; -tea, Pai.; te, Si. Pon.; -ee,15 Si. Win.;du, Tling.; tuk, M. Choe.; Le, Wiy.

57. Plural; ūu, Siusl.; -ya, H. Po.; -i, Y. Yu.; -ī (reflexive plural) -i, P. Yok.; -yu, Ath. Hu.

58. Plural; -e, H. Ka.; -s, Y. Wap.; -c, Chin.; -s, Iro.

59. Plural distributive; -tx, Siusl; -t, H. Sal.; -da, H. Was.; ta-, Wiy.; it, Tak.; -tH, Sa.; de-, Tsim.; t-, Chin.; -to, P. Mai.; te-, Ath. Hu.; -tï, Pai.; -t-, Si. Bil.; t'e, Si. Pon.; tak, Maya; -d (in verbs), Al. Ojib.; -ta, Cad.; -te-, Y. Wap.

60. Plural; -el, -l, H. Sal.; l-, Tsim.; -l, Ath. Hu.; -le, Y. Wap.; -n, Al. Ojiff?

61. Plural; -me, P. Cost.; -ma, H. Po.; -Em, Kwak.; -ma, Chin.; -mï, Pai.; -ma (plural animate objeet), Si. Pon.; -pi, Si. Dak.16

62. Plural; -ke, P. Miw.; -k, H. Po.; -kie, H. Was.; -uks, Sa.; -k', Kwak.; -ike, uks, Chin.; -kī,-k, Ath. Ka.; -qan, -ik, Kut.; -qa, Pai.; ga-, Tsim.; ge, Si. Pon.; -ke, Al. Ojib.; -ka, Cad.; -ke, Iro.

63. Passive prefix; t-, Siusl.; t-, H. Sal.; do-, H. Po.; te-, Y. Wap.; ad-, Atl. Hu.; t'a-, Si. Dak.; -ta (?), Cad.

64. Passive prefix; m-, Wiy.; me-, Y. Wap.; m-, Si. Dak.

65. Potential; -ni, P. Miw.; P. Mai.; -ni, Si. Dak.; -uñ, Ath. Ka.; -ni, Nah.; -ni, Zap.

66. Present tense; -n, Siusl.; -ya, Y. Wap.; -i, H. Was.; -a, H. Po.; -yi, Pai.

67. Quiekly; dji-, Tling.; djin, Si. Win.

68. Reflexive; -po, P. Miw.; -pu, -mu, P. Cost.; -muxu (reeiprocal), mai', Y. Wap.; -vani, H. Ka.; -ap' (reciprocal), Kwak.; -me'k, Kut. ; vï, Pai.; ma-, Nal.; -wa-, -pa-, Si. Win.; -wi, Wiy.; wi-ti, Cad., ib, Maya.

69. Reflexive; -an, Tak.; -naw (reeiproeal), Siusl.; na-, Tsim.; n-, Pai.; d-, r-, Si. Win.

70. Subordinating suffix; -ye, Ath. (Nadene ge); -ri, Tling.; -ga, Si. Win.; -qa, Pai.; -gun, H. Ya.; gu-, Wiy.; -x, Kwak.

71. Suffix indicating "times", for numerals; -ita, Siusl.; -ta, Atl. Ka.; -ta, Y. Wap.; -yit, Kut.; -ta, Pai.

72. To; -t, H. Sal.; -d-, Tak.; -t-, Clin.; -tu, Y. Wap.; -ta, Si. Dak.; -d, Nadẻne (S); -tu, Cad.; t-, Iro.

14 On Frachtenberg's authority.

15 Winnebago e corresponds to Siouan te.

16 Dakota $\mathrm{p}$ goes back to Siouan *m. 
73. Transitive suffix; -t, Co.; -d-, Tak.; -t-, Y. Wap.; -d, Tsim.; -L-, Ath. Hu.; -ta, Si. Dak.; -du (?), H. Po. (R) ; -d-, Kwak.; -t-, (q) Al. Fox.

74. With; -ti, Y. Wap.; de, Tsin.; tîn, Hai.; -L, Ath. Hu.; -ni, Zap. (R); -ne, Iro.

75. Verbalizing suffix; -āi, Siusl.; -e, H. Po.; -a, Kwak.

\section{NOUNS}

1. Arrow; na-tses, Atl. Hu.; zi-e, Ath. Mon.; tsapi, Wiy.; sa'a, H. Chim.; tsu, H. Po.; me-tse, Y. Wap.; tee-mo, yatei, P.; teoar, Sa.; atūs, Al. Cree; tald-slii,17 Lut. Kla.; ts'i-talen, Hai.; han-tlem, Kwak.; ts'-hate, Nootka; tlok, tats'omen, Sa.; os-ki (?), M. Choc.

2. Belly; bu-s, P.; bû-t, Ath. Be. ${ }^{18}$ bunu, S. Hopi ; ben, Tsim.; iffu-ka, M. Choc.; miss-ad, Al. Ojib.

k'en, Sa.; ik-pi, Si. Dak.; ika, H. Sal.; k'ita, Y. Wap.; nka-sh, Lut. Kla.

3. Bird; teil-teil, P.; dila, H. Chim.; tsita, H. Po.; tsitsa, Y. Wap.; izitka-dan, Si. Dak.; detta-ni, Ath. Mon.; hushi, M. Choc.; teite, Maya; totli, Tling.; tsōwots, Tsim.; ts'ekō, Kwak.; tsuts-kic, Wiy.; k-ontities, Iro.;19 teika-sh, Lut. Kla.

4. Blood; sak, sede, P.; sitsö, H. Chim.; ez, ödj, Uto-Aztecan; tcheke-lli, Lut. Kla.; ci, Tling.; sîH, Sa.; issish, M. Choc.; $a^{\mathrm{n}} \mathrm{e}, \mathrm{Y} . \mathrm{Yu}$;

te-l, Ath. Mon.; itle, Tsim.; wa-do (?), Al. Ojib.

kue-tl, Sa.; gai, Hai.; kawi-k, Wiy.

uñwe, S. Hopi ; wan-mo (q), Kut.; we, Si. Dak.

5. Bone; te'ei, ts'ix, P.; tsita, Y. Wap.; hu-txun, H. Chim.; ts'un-ne, Ath. Be.; sâyu-p(?), cīao, Sa.

iaqa, S. Hopi ; a'o, S. Gitanemuk; o'o, Pai.; qak, Kwak.; k`ō-kōtl,20 Sa.; ka-n, Al. Ojib.; kako, Lut Kla.; xau-cin, Sa.21

6. Bow; tl.k'et, Hai.; hau-ktak, Tsim.; tlkues, Kwak. ; k'tsēite, tâqoats, łaq’u, Sa.

t’āo, Kut.; dayap, lawan, P.; atchabiy, Al. Cree; auta, ats, S.; t'i-n, Ath. Mon.; ita-zipa, Si. Dak.; nte-ish, Lut. Kla.

7. Boy, man; xai, Ath. Hu.; kowi, Wiy.; kawi, H. Po.; k'єo, Y. Wap.; k'aina, koteo, P.; kwi, Ath. Mon.; qea, kwiti, S.; gyi-t, Hai.; qapqo,22 Kwak.; ak'un, Maya; kwiu-säns, ${ }^{23}$, Al. Ojib.

tei, H. Po.; teaki, Lut. Kla.; wi-ta, P.; iōot, Tsim.; tcōi, tuot, Sa.; staha-tl, Kut.; ath, Nootka; hotcin, S. Win.; hata-k, M. Choc.

8. Brother (elder); guāi, Tling.; wegy, Tsim.; gyīi, Kwak.; k•oa-lm, k`atck, Sa.; ki, Iro.

ta-ka,24 P. Mut.; ito-l, H. Po.; etche-le, Ath. Mon.; teinye, Si. Dak.

9. Canoe, boat; yauk, Tling.; -qsa, Tsim.; -qs, Kwak.; qu-tl, Sa.; keye, Y. Wap.; kula, M. Choc.

tlōu, Hai.; ttsi, Ath. Mon.; yak-tsomitl, (q) Kut.; towi, H. Sal.; wa-ta, Si. Dak.; tei-man, Al. Ojib. (-man is a nominal suffix).

10. Cold; sīat, Tling.; ts'ât-it, Sa.; atho, Iro. ; ts'at-el, H. Sal.; *axate‘a, H. (S) ; edza, Ath. Mon.; sıni, Si. Win.; ze, Nah.; hoteuk-wa, M. Choc.

ska, Lut. Kla.; quī, Hai.; qkuatko, Tsim.;25 qäi-tl, qE-tl, Sa.; skāt'ei, Kut.; ge-ts, Wiy.; ka-dj, Al. Ojib.

\footnotetext{
17 Shi is a nominal suffix.

18 Isolated by mýself.

${ }^{19} \mathrm{~K}$ - is a nominal prefix corresponding to Algonkin gi-, Kutenai, aq-

20 Reduplicated stem.

21 cin is a noun ending.

22 Reduplicated stem.

23 -säns is a diminutive.

$24 \mathrm{ka}$ is a noun suffix isolated by myself.

${ }^{25}$ Reduplicated stem.
} 
11. Deer, elk; tee, Si. Win.; thez-il, Ath. Mon.; teisk, Tling.; sîn, Tsim.; tlols, Kwak.; shua-i, Lut. Kla.; tlales, Sa.;26 tante, P. Win.;27 tso-toko, Y. Wap.; se, Maya; isi, M. Choc.; bi-ce (?), H. Po.

12. Ear; oteo, P. Cost.; ea-m,28 H. Chim.; atoa, Kwak.; tawa-k, Al. Ojib.; ahonta, Iro.; tl'ā-na, Sa.; edza, Ath. Mon.; tse-ma, Y. Wap.

gyū, Hai.; k'oā'a-na, Sa.; g'o-koat,29 Kut.; hak-lo, M. Choc.;30 nakaz, Nah.; nore, Si. Dak.

13. Eye; hin, eo, se, sa, P.; sot, H. Chim.; ui, huui, H. Po.; isi, ix, Uto-Aztecan; ie-ta, Si. Dak.; eliiluk, (?) M. Choc.; hutsi, Y. Wap.; hu-l, Y. Yu.; ite, Maya; osh-kinji, (?) Al. Ojib.; ena * $^{*}$ eta (?), Ath. Mon.

14. Fire; xon, kon, Ath. Be.; qu, Pai, ru, Si. Dak.; ho, xo, H. Po.; haiuk, Sa.; ki-nk $\mathbf{k}^{\circ}{ }^{\prime} k^{\circ} 0,31$, Kut.; ishkote, Al. Ojib.; he-l, Y. Wap.; sa (१), P. Mai.

lak, Tsim.; luak, M. Choc.; inik, Nootka; nēiq, Sa.

tle, Nah.; ito, M. Choc.; da-, Si.; tewi, Lut. Kla.

apu, H. Chim.; pe-te, S. Win.; pe-tl, Nah.; me-s, Wiy.

15. Foot; ko, P.; ke', Ath. Be.; hokya, Si. Hopi; ka-ma, H. Po.; ko-kue,32 Kwak.; sqa-n, Sa.; teka-tc, Wiy.; oka-d, Al. Ojib.; ka-s, Cad.

ti, P. Cost.; tea-de, Ath. Be.; ontsa (knee), Iro.; tsaka, S. Gitanemuk; teuk-sh, Lut. Kla.; si, Tsim.; dji-ein, Sa. ; sak, Kut.; siha, Si. Dak.; osi-d, Al. Ojib.; us, Cad. asi, Iro.

hupo, P.; pe, Y. Wap.; pet-cl, Lut. Kla.

16. Girl, woman; tea'e, Y. Wap.; atsya, H. Po.; ttse, Ath. Mon.; eätq, Tling.; ista, Iro.; silua-tl, Nah.; iskwe, Al Cree; shiwa-ga, Lut. Kla.; wi-tein, Si. Dak.; dja, Hai.; tsetaq,34 tlotsma, Nootka; sā.tltq, ciāktee,34 Sa.; ōtē (?), Kut.; wa-teer, Wiy.

17. Hair; ana, P.; hin, Si. Win.; hima, H. Chim.; hini, S. Hopi; e, hee, H. Po.; haiHte, Kwak.; hishi, M. Choe.; oshkin-jig, Al. Ojib.

ethi, Ath. Mon.; ma-ne (?), Lut. Kla.

18. Hand; isk, P.; ea (arm), H. Po.; sia-pe (?), Kwak.; shak-ba (?), M. Choe.; a $>{ }^{*}$ sa (?) (arm), Si. Dak.

hita, H. Chim.; la', Ath. Be.; tana, H. Po.; djin, Tling.; odja, Sa.; otchi-tji, Al. Ojib.; 'odja, t’ä-lō, Sa.; an'on(?), Tsim.; la-ka (arm), Y. Wap.; na-pe, Si. Dak.; ne-p (q), Lut. Kla.

uku, P.; kilн, aqa-n, Sa.; gèi, Kut.

mo, Pai.; ma, Tanoan; me, Y. Wap.; ibba-k, M. Choc.; mā, P. Mai.; we-s, Wiy.

19. Head; to-l, tco-l, P.; tco-, Pai.; tsī’ Ath. Be.; ei-na, xi-ya, H. Po.; hi-ma, H. Chim.; sāia (face), Kwak.; tsa-l (face), Tsim.; cti-gwan, Al. Ojib.; teli-sh, Lut. Kla.

moo-c, Sa.; emē (face), Kwak; pa, Si. Dak.; an-umpa (?), M. Choc.; po-l, Maya ; ma-l, Huave; ba-L (hair), Wiy.; pu-ks, Cad.

26 Reduplicated stem.

27 Reduplicated stem.

28 Noun suffix.

29 Reduplicated stem.

30 -lo is probably a noun suffix.

31 Reduplicated stem.

32 Reduplicated stem.

33 Reduplicated stem.

34 Reduplicated stem. 
20. Hill; domi-t, P.; tepe-tl, Nah.; mual, paau’a, S.; paiyi, H. Po.; mə-ta, Y. Wap.; paha, Si. Dak.; bo-kko, M. Choc.; s-mant, Sa.; awu, H. Chi.; p'o, Tanoan.

teo-L, P.; wa-djiw, Al. Ojib.; 'edje,, Ath. Be.

s-qan-est (?) Tsim.; g'o-kwis, Kwak.; s-kum, Sa.; xi-s, Atl.. Ka.; cia, Tling.; xe, Si. Win.

21. Iee; teara, Si. Dak.; t'ek, Tling.; ot'en, Ath. Mon.; dāu, Tsim.; tl'oq, Kwak.; tauō, Sa.; ok-ti (q), M. Choc.

k.al-ga, Hai.; gut, Kut.; s-qoi-nt, Sa.; k.ōuq, Kwak.

22. Land, earth; ama, H.; oma, Y. Wap.; ma, Si. ; mëi, Sa.

tsuwut, Pai.; dzāatseks, 35 Tsim.; tso, Y. Wap.; otle-s, Ath. Mon.

tlga, Hai.; t’ekya, Kwak.; kai-la, Lut. Kla.; ak`i, Al Ojib.

23. Liver; huei, H. Chim.; zû-t, Ath. Be.; ea-la, H. Po.; salla-kha, M. Choc.; hon (q), Y. Yu.

ku-lla, P. Miw.; ko-k, Y. Wap.; ko-n, Al. Ojib.

24. Moon; op, P.; mö-yawa, S. Hopi ; pi (sun), Si. Dak.; beo, Zap.; p'o, Tanoan; poo, Mixe (R); po, Maya; shpa-sh, Lut. Kla.

25. Moceasin; titl, Tling.; ts'â-oqs, Tsim.; tlek-ein, Sa.; tlan, Kut.

k'e, Ath. Mon.; k'ena-q, Kwak.; k'en-q, Sa.

26. Mouth; ca-ma, ce-ma, P.; shu-m, Lut. Kla.; hawa, H. Chim.; ha, habo, H. Po.; za', Ath. Be.; osa, Iro.; su-ms, Kwak.; so, Tanoan (R); ju, tsua, Zap. (R); odo-n (q), Al. Ojib.; tsu-tsa, sâ-sen,36 Sa.; wi-teai, Si. Dak.; ita-kha, M. Choe.

k'a, Tling.; qē-tl'a, Hai.; ku-tl'aq, Tsim.; k'a-tluma (?), Kut.; akau, Cad.; ha, Maya, a, Zap. (R), i, Si. Dak.

moa, Pai.; apa, M. Choc.

27. Nail; soki, Pai.; sakta, Lut. Kla.; tleqs, Tsim.; tsEm-tsem, Kwak.; te'atlte'a,37 Nootka; atakal, M. Choe.; ete, H. Po.; ti, pitei, P. Miw.; teu-e, Y. Wap.; tu-s, Ath. Mon.

kus, Y. Yu.; gōu-kp, Sa.; okatan, Si. Dak.; me-tkan, Wiy.; oshkanj, Al. Ojib.

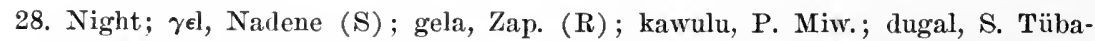
tulabal; ka'was, Si. Win.

diwe, duwe, H. Chim.; tibi-k, uteuwa, Y. Wap.; uteu, Si. Catawba. lıanhe, Si. Win.; hime, H.

29. Nose; huk, sin, son, P.; hoxu, H. Chim.; ju, Zap. (R) ; ds'ak, Tsim.; ni-ts'a,

Kwak.; odja-ni, Al. Ojib.; teos, Cad.; p-shi-sh, Lut. Kla.

kun, Hai.; kuk-tsatla (q), Kut.; oski-wan, Al. Cree; equ, Sa.

la, H. Po.; tlo, Tling.; tere, Al. Wiy.

won, Ath. Be.; pore, Si. Dak.; maq-sin, Sa.

30. Old, old person; itrine-ulla, H. Chim.; cōn, Ath. Be.; e'a-k, Si. Win.; t'sehi-ka,

Lut. Kla.; chikki, M. Choe.; bu-sa, bu-tceki, H. Po.; ki-tei, Al. Ojib.; akaio-n (?), Iro.

31. Rain; upa, P. Win.; ma-k, Y. Wap.; wa-s, Tsim.; mi-tla (?), Nootka; umba,

M. Choc.; mara, Si. Dak.; gi-miwan, Al. Ojib.; we-sh (ice), Lut. Kla. sēu, Tling.; tee, H. Po.; tcīe-tl, Sa.; shi-t, Ath. Mon.

${ }_{35}$ Reduplicated stem.

36 Reduplicated stems.

37 Reduplicated stem. 
32. Sleep; po, H. Chinı.; bû-l, Ath. Be.; ni-ba, Al. Ojib.; apui, Pai.; me-qet (?), Kwak.; pel-et, Sa.

k’'a, Hai.; qstoq(?), Tsim.; gy’ā-tla, Kwak.; g•’onl, Kut.; ktana(?), Kut.; k'ana, xin, P. Win.

sim, H. Po.; in, Y. Yu.; *tean, Si.; tsi-toma, tlä-tsit, etut, itq, Sa.

33. Snake; wa-kan, Si. Win.; coa-tl, Nalı.; kan, Maya; gine-big, Al. Ojib.; ołkai, Sa.; wi-shink (?), Lut. Kla.

34. Snow; p’a, H. Ya.; pi-l, Y. Wap.; mād-em, Tsim.; mā.k’a, Sa.; me, Al. Fox; wa, Si. Dak.

iōkoa, Kwak.; kn’ai, Sa.; ke-sh, Lut. Kla.; ktlō, Kut.

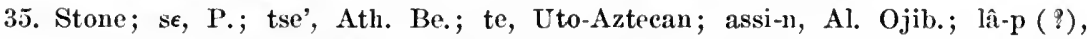
Tsim.; t'e-sem, Kwak.; tla-tsa, Htlō-t, Sa.; ta-li, M. Choc.; le-l, Y. Wap. kaa, H. Chim.; tlqa, Tling.; k’E-tla, Sa.; kta-ti, Lut. Kla.; ku, Tanoan; kul, Zap.

36. Sun; sa-s, P.; asi (today), H. Chim.; ca', sa, Atl. Be.; sE-n, Hai.; sa, Tsim.; gi-siss, Al. Ojib.; hashi, M. Choe.

da, H. Po.; alla, H. Chim.; dawa, S. Hopi; nala, Kwak.; te-gyem (?), Sa.

37. Tongue; èthu, Ath. Mon.; tsū-di, Ath. Be.; teezi, Si. Dak.; tl’ōt, Tling.; te'up, Nootka; tīH-tsa, tā-tla, tix-usal, Sa.; dūe-la, Tsim.; wa-tlōnak (?), Kut.; isun-lash, M. Choe.

leñi, S. Hopi; leti-p, P.; dena'-ni, Al. Ojib.; ennasa (q), Iro.

38. Tootl ; si, sit, P.; hutsu, H. Po.; da-ma(?), S. Hopi; tu-t, Lut. Kla.; dzeñ,

Hai.; sia, Kwak.; dji-nis, dji-dis, itsa, Sa.; co, Maya; hi(q), Si. Dak. $\bar{o} q$, Tling; gyi-ky, Kwak.; ku-nan(q), Kut.; o, H. Po.; ua-n(?), Tsim. Hwe, Kwak.; wō', Ath. Be.; m-ept, Wiy.; bi-d, Al. Ojib.

39. Trail; inu, P. Cost.; na, Ath. Hu.; nan, Pai.; nan-gu,38 Si. Dak.; stu, Lut. Kla. pö, Pai.; po, Tanoan; p'o, Zap. (R); bo, P. Mai.; bej, Maya; mik-an, Al. Ojib.

40. Tree, wood; tsa, teu-s, P.; teû-t, Ath. Be.; djā', Sa.; atsa, H. Chim.; tean, Si. Dak.; iti, M. Choc.

k•an, Tsim.; g’an, Tling.; kum-tl, k'oi-q, koū-s, Sa.; hai, ka-le, H. Po.; an-ku, Lut. Kla.

41. Water (ef. also to drink); aka, H. Chim.; oka, M. Choe.

pa, Nah.; p'o, Tanoan; mu, Zap. (R) ; me-m, P.; wou, Kut.; pō (fluid), Al. Fox; ni-bi, Al. Ojib.; ampu, Lut. Kla.; waa-n, Kwak.; mei, Y. Wap.; awe-n, Iro.

42. White; l-kai, Ath. Hu.; ka-le, H. Po.; k'aie-l, Y. Wap.; kaiya, ko, ko-le, P.; s-ka, Si. Win.; ts-q, Sa.; cai, ce, ieta, S. mâks, Tsim.; pॄk, Sa.; pal-pal, Lut. Kla.

\section{VERBS}

1. to be; -ka, Si. Win.; -qa, Pai.; -ke, Y. Wap.; g'o, P. Yok.; 'a $>^{*}$ ga, Ath. Ka.; k•em, H. Po.; gi, Tsim.; -ki, Kwak.; -e (?), Chin.; -qa, Kut.; kia, M. Choe.; -ka, Al. Ojib.; koh, Mixe.

2. to bite; -gal, H. Ya.; g'a (with teeth), H. Po.; k'a, Y. Wap.; g'ai, Tsim.; ka*w, P. Cost.; gan-e, Chin.; qa-s, Kut.; gayaw (q), Tak.; kis-li, M. Choe.; kª̈ (to eat), Sa.; ku, Maya; rawa, Zap.; qua (to eat), Nah.; ka, Lut. Kla.; ike-ks, Iro.; ‘k, Kut.; kai, Mixe; ya (Nadene ga?), Ath. Ka.; g, Kwak.; ka-ts, Cad.

${ }^{38} \mathrm{gu}$ is a suffix; nan goes back to tean. 
3. to come; to come in ; g•âo, Tsim.; g•ax, Kwak. ; klēe-k, Sa.; qo, Kut.; ak, Wiy.; gaio-n, Iro.

tco, Si. Win.; ho-teo, Y. Wap.; t, Chin.

4. to cry; ra-k, Si. Win.; yara, Pai.; k•a-m, Y. Wap.; ixu-ka, P. Cost.; xe (to sing); xa-xs, Tsim.; kwe, Kwak.; k'oa-kt, Sa.

5. to do; un, Si. Win.; uni, Pai.; in, P. Cost.; uni, Zap. (R).

k`am, Y. Wap.; k'an, Tsim.; k'eme, Tak.; konni-s, Iro.

it, Kut.; eta', H. Sal.; ato-łi, M. Choc. ; tar, Cad.

ax, Kwak, x, Chin.; aki, Wiy.

6. to be dead; lal-ok, Y. Wap.; loho, Tak.; lia, H. Po.; łє’ł, kwak; illi, M. Choc.

7. To drink; g'oki, H. Po.; aks (water), Tsim.; nāq(q), Kwak.; k•oāi, Sa.; qa-mst, Chin.; uuk, Mixe; i', u'-(?), Zap. (R); uk', Tak.; -ku (water),

Kut.; oka (water), M. Choc.; nax-gan, Si. Win.; hik, S. Hopi;.uk’i, Y. Wap.; uk, P.; u'k, Maya.

8. to eat; ama, H. Sa.; ham, Kwak.; apa, M. Choc.; pa, Y. Wap.; mak, Maya; mei, Mixe; am, P. Yok.; maL, Wiy.; amw, Al Fox.

ta, Si. Dak.; -tse, Y. Wap.; duy, P. Yok.; tan, Ath. Ka.; to', Zap. (R); tzu-tz, Mixe; dza, Tsim.

9. to give; ama, M. Choc.; me, Y. Wap.; ma, P.; ma-gi, S.; lramat, Kut.; mak, H. Sal.; mī, Al Fox.; wi, Iro.

10. To give; tsew, Wiy.; diaxa, H. Po.; toxi, H. Sal.; tea-ni, Y. Yu.; doyu, P.; de, djE-t, Tling.; etei (give), M. Choc.; sa-teit (?), Sa.; itu-ran, Si. Dak. gyen-am, Tsim.; qūitsh-it, Sa.; ku, Si. Win., Dak.

11. to go; ye, re, le, Si.; le, Y. Wap.; 'a, H. Chim.; ne, H. Po.; a'l, Wiy.; ya, Al Fox; yao, ie, Tsim.; ì, Chin.; la, Kwak.; ì, Iro.;nä-c, Sa.; īa, M. Choc.; $\theta$ a, tsa, Zap. (R); yoi, Mixe; at, Tling.; ut, Cad.; yana, Tak.; ina, M. Choc.; tan, P. Yok.

12. to kill; t'e, Si. Win.; tû-k, Ath. Ka.; ute', Zap. (R); t'a, W. Wap.; dza-k, Tsim.; du-, eik-En, tl'ek'-un, Sa.; li-k, P. Cost.; ea-k, H. Po.; t'ia, Hai.; dja-k, Tling.

13. to laugh; ikea, H. Chim.; ke, kuwai, H. Po.; ken, hai, P.; k'a, Hai.; hikeai-t'e, Si. Win.; qā-aqiam,39 qoi-qoayos, Sa.; yuk-pa (?), M. Choc.

14. to run; aga, H. Po.; g•ô-l, Tsim.; ngo, Chin.; gu-ka, Lut. Kla.; kuw, Y. Wap.; re-d, Nadene (S); hog, Tak.; kuwa, Si. Dak. nuxu, Kut.; nugi, Pai.; noq, Kwak.

pulu, Y. Wap.; poya, Pai.; paya, P. Cost.; hiwilìu', Tak.; pìli (go), H. Po.; bate-li, M. Choc.

15. to see; ta, Si. Dak.; ts'e, H. Sal.; do-qu, Kwak.; äts-qfm, Sa.; chaa-n, Maya; nao, Y. Wap.; iñ, Ath. Ka.; ni, Tsim.; ahni, M. Choc.; inu, P. Cost.; nä-, Al. Fox.

öka, P. Cost.; ga-r, H. Po.; kel, Chin.; keye (observe); ge’, Ath. Ka.; xeL (look for), Wiy.; ikke-ns, Iro.

16. to sing; tai', Y. Wap.; udü-k, P. Yok.; -le', Ath. Ka.; elt-um, Sa.; ola, M. Choc.; ila, Zap. (R).

17. to sit; dai, Ath. Hu.; d'a, Tsim.; te-m, Wiy.; tei-t, tea-teiu, dja-tei, H. Po.; cu, Y. Yu.; otcu, teawa, xo, P.; ya-teo, yan-dji, S.; tsi-am, Sa.; ansha,

M. Choc.; ną-k $>{ }^{*}$ tcan-k, Si. Win. k"aua, Hai.; gua, Kwak.; gati, S.

39 Reduplicated stems. 
18. to speak; na-t'e, Tak.; t'a, Maya ; tsxa, Kut.; de-de, H. Po.; da-lx, Tsim.; tcu-t, Sa.; ne, Zap.; uaan, Mixe; 'hi-t'e, Si. Win.; te, Al. Fox.

a, M. Choc.; ha, Si. Win.; ai-ngu, Pai.; ha, Y. Wap.; xay, P. Yok.; hao, Tsim.; xa, Kwak.; ke (?), Kut.; hi, Al Fox; a, Iro.

19. to split; da-k, H. Po.; ts'â, Tsim.; ts'Et, Kwak.; tea'є, Y. Wap.; at, P. Cost.; xa-its'iwi, Tak.; tsik, Kut.; chu-ła, M. Choc.

20. to stand; dano, Wiy.; dji-tcon, H. Po.; tö-s, ta-la, it-ma, P.; tla, Kwak.; tetli-qa, t'öwe-s, Sa.; hayit-k, Tsim.; te, Si.; -d-, Iro.; ya, yen, Atl. Hu.; ya-l, H. Was.; yan, Y. Yu.

gya, Tling.; e-kät, Sa.; gawi-ska, Kut.; hika, M. Choe.

21. to step; t'oq, T'sim.; t'a, Y. Wap.; tal, Ath. Ka.; t'e-pa, Kwak.; teaede, Si. Dak.; da (to jump), H. Po.; day, P. Yok.; haton-chi40 (to jump), M. Choc.; La-l, Wiy.

22. to strike; tok, P. Miw.; -ti, Y. Wap.; te-gis,41 Si. Win.; doc, H. Po.; L'eq, Chin.; săag, Tak.; te, Zap. (R); t'a-tk, Iro.

23. to talk; haqa-l, Kut.; ka-n, Maya; koia, Mixe; hi-ge, Si. Win.; qa (sing) Pai.; ba-qo, H. Po.; ka-l, Y. Wap.; ko, P. Cost.; 'a, Ath. Ka.; k’ōai, Sa.; kuL, Chin.; ka-n, Al. Fox.

24. to wait; pe, Si. Win.; pihe, Y. Wap.; eme-ni, P. Cost.; būo, Tsim.; k-pa, Kut.

25. to wash; tea, Y. Wap.; ita, P. Cost.; de', Atl. Ka.; teawa-te, H. Sal.; ts'ał-ksen (q) (rinse out), Tsim.; ts'e-xu, Kwak.; tzä-wan, Sa.; tseaya-ga'n, Tak.; tse-k, Kut.; ache-fa(?), M. Choc.; tsiu, Mixe; tcai, H. Chim.

\section{PERSONAL PRONOUNS}

1. I; ka-min,42 Kut.; ka-nni,43 P. Miw.; xa, Tling; -k, Yur.; 'ke, H. Sal. ha, H. Po.; ha, Y. Wap.; ha, Si. Win.; ł, Hai.; he, H. Sal. sa, M. Choc.; ci, Ath.; -s, P. Mai.; -ts, Sa.

3. We; tu, Tling.; t'al-Añ, Hai.; dep, Tsim.; itamo, Sa.; nteai, Chin.; te-ne, H. Sal.; il, M. Choc.; -ta-, Nah.; -t-, Zap.; itl, Sa.; at, Wiy.; hi, hin (?), Si. Win.

2. I; an, M. Choc.; n, Tsim.; -En, Kwak.; nö, S.; nai-ka, Chin.; ni, P. Mai; ne, hi, Si. Win.; n, Zap.; n, Mixe; n, Maya; -n-, Al.

4. Thou; ni-nko, Kut.; -no, P. Mai.; n, Ath.; ne, S.; n, H. Sal.; -no, S.

5. Thou; is, tci, M. Choc.; -Es, Kwak.; -s-, Iro.; -s, P. Miw.; e-, Si. Win.; -ts, Yur.; -s-, Cad.

6. Thou; mi, P.; ma, H. Po.; mi, Yu.; m, Tsim.; öm, S.; mu, H. Sal.; mai-ka, Chin.; m, Mixe, -m, Yur.

7. Thou; da, Tling.; r-, 1-, d-, Si.; t-, Nah.; t, Maya; r-, Huave; -r, -l, -d, Zap.; -it, Wiy.

40 chi is the causative.

41 gis is a suffix indicating aspect.

42 Probably a pronominal ending.

43 Pronominal suffix. 


\section{DEMONSTRATIVES44}

1. itü, S.; ta, P.; di, Nadene; du, Tsim.; de, Si.; te, Y.; t’ai-tl, Sa.; at, M.; to, Iro.

2. igi, S.; ka, P.; ye $>^{*}$ gai, Nadene; gu, Tsim.; -k, Chin.; ga, Si.; gya, Kwak.; aqa, Sa.; ak, M.; ku-, H. Po.; gu, Wiy.

3. pe, bami, S.; mě, Nadene; me, Si.; we, Y.; milı, M.; me, H. Po.

4. xe, P.; ha, Nadene; i, Iro.; x, Chin.; he, Si.; he, Y.; he, Kwak.; hëi-tl, Sa..; ho, M.; he, H.

\section{NUMERALS}

1. Three; dlku-nutl, Tling.; tlēq, Sa.; tolokot. P.; rik, Wiy. xats, teas, H.; k’atstsa, Nootka; g•atlsa, Kut.; guant, Tsim.; siātla, Sa.; team-ni, Si. Dak.; tuteina, M. Choc.; ndani, Lut. Kla.; asen, Iro.; n-iswi, Al. Ojib.

subu, sibo, H.; mo-l, Y. Yu.; la-pai, sa-pu, P.; pahi, S.

2. Four; diñ-k, Tling.; riaw, Wiy.; ta-k, Ath. Hu.; tsöye, uti-t, ka-tuac, P.; ki-tea, H. Yuman; watca, tea-pap, S.; djop, Si. Win.; ushta (\&), M. Choc. tqa-lpq (?), Tsim.; qōs-ěna, Sa.; qātsa, Kut. mō, Kwak.; mōs, Sa.

44 This, that.

Transmitted, March 26, 1919. 




\section{UNIVERSITY OF CALIFORNIA PUBLICATIONS - (CONTINUED)}

Vol. 7. 1. The Emeryville Shellmound, by Max Uhle. Pp. 1-106, plates 1-12, with 38 text figures.' June, 1907

2. Recent Investigations bearing upon the Question of the Occurrence of Neocene Man in the Auxiferous Gravels of California, by William $\mathbf{J}$. Sinclair. Pp. 107-130, plates 13-14. February, 1908

3. Pomo Indian Basketry, by S. A. Barrett. Pp. 133-306, plates 15-30, 231 text figures: December, 1908

4. Shellmounds of the San Francisco Bay Region, by N. C. Nelson. Pp. 309356, plates 32-34. December, 1909

5. The Ellis Landing Shellmound, by N. C. Nelson. Pp. 357-426, plates 36-50. April, 1910

Index, pp. 427-443.

Vol.8. 1. A Mission Record of the California Indians, from a Manuscript in the Bancroft Library, by A. L. Kroeber. Pp. 1-27. May, 1908 ......................

2. The Ethnography of the Cahuilla Indians, by A. L. Kroeber. Pp. 29-68, plates 1-15. July, 1908

3. The Religion of the Iuiseño and Diegueño Indians of Southern California, by Constance Goddard Dubois. Pp. 69-186, plates 16-19. June, 1908 .......

4. The Culture of the Luiseño Indians, by Philip Stedman Sparkman. Pp. 187 234, plate 20. August, 1908

5. Notes on Shoshonean Dialects of Southern California, by A. L. Kroeber. Pp. 235-269. September, 1909

6. The Religlous Practices of the Diegueño Indians, by T. T. Waterman., Pp. 271-358, plates 21-28. March, 1910

Index, pp. 359-369.

Vol.9. 1. Yana Texts, by Edward Sapir, together with Yana Myths collected by Roland B. Dixon. Pp. 1-235. February, 1910

2. The Chumash and Costanoan Languages, by A. L. Kroeber. Pp. 237-271. November, 1910

3. The Languages of the Coast of California North of San Francisco, by A. I. Kroeber. Pp. 273-435, and map. April, 1911 Index, pp. 437-439.

Vol. 10. 1. Phonetic Constituents of the Native Ianguages of California, by A. I. Kroeber. Pp. 1-12. May, 1911

2. The Phonetic Elements of the Northern Paiute Language, by T. T. Waterman. Pp. 13-44, plates 1-5. November, 1911

3. Phonetic Elements of the Mohave Language, by A. I. Kroeber. Pp. 45-96, plates 6-20. November, 1911

4. The Ethnology of the Salinan Indians, by J. Alden Mason. Pp. 97-240, plates 21-37. December, 1912

5. Papago Verb Stems, by Juan Dolores. Pp. 241-263. August, 1913 .............

6. Notes on the Chilula Indians of Northwestern California, by Pliny Earle Goddard. Pp. 265-288, plates 38-41: April, 1914

7. Chilula Texts, by Pliny Earle Goddard. Pp. 289-379. November, 1914 Index, pp. 381-385.

Vol. 11. 1. Elements of the Kato Language, by Pliny Earle Goddard. Pp. 1-176, plates 1-45. October, 1912

2. Phonetic Elements of the Diegueño Language, by A. L. Kroeber and J. P. Harrington. Pp. 177-188. April, 1914

3. Sarsi Texts, by Pliny Earle Goddard. Pp. 189-277. February, 1915

4. Serian, Tequistlatecan, and Hokan, by A. L. Kroeber. Pp. 279-290. February, 1915

5. Dichotomous Social Organization in South Central California, by Edward Winslow Glfford. Pp. 291-296. February, 1916

6. The Delineation of the Day-Signs in the Aztec Manuscripts, by T. T. Waterman. Pp. 297-398. March, 1916

7. The Mutsun Dialect of Costanoan Based on the Vocabulary of De la Cuesta, by J. Alden Mason. Pp. 399-472. 'March, 1916 Index, pp. 473-479. 
UNIVERSITY OF CALIFORNIA PUBLICATIONS - (CONTINUED)

Vol. 12. 1. Composition of California Shellmounds, by Edward Winslow Gifford. Pp. 1-29. February, 1916

2. California Place Names of Indian Origin, by A. I. Kroeber. Pp. 31-69. June, 1916

3. Arapaho Dialects, by A. I. Kroeber. Pp. 71-138. June, 1916

4. Miwok Moieties, by Edward Winslow Gifford. Pp. 139-194. June, 1916 ...

5. On Plotting the Inflections of the Voice, by Cornelius B. Bradley. Pp. 195218, plates 1-5. October, 1916

6. Tíbatulabal and Kawaiisu Kinship Terms, by Edward Winslow Gifford. Pp. 219-248. February, 1917

7. Bandelier's Contribution to the Study of Anciens Mexican Social Organization, by T. T. Waterman. Pp. 249-282. February, 1917

8. Miwok Myths, by Edward Winslow Gifford. Pp. 283-338, plate 6. May, 1917

9. California Kinship Systems, A. L. Kroeber. Pp. 339-396. May, 1917

10. Ceremonies of the Pomo Indians, by S. A. Barrett. Pp. 397-441, 8 textfigures. : July, 1917

11. Pomo Bear Dectors, by S. A. Barrett. Pp. 443-465, plate 7. July, 1917 Index, pp. 467-473. Vol. 13. 1. The Position of Yana in the Hokan Stock; by E. Sapir. Pp 1-34. July,

2. The Yana Indians, by T. T. Waterman. Pp. 35-102, plates 1-20. February, 1918

3. Yahi Archery, by Saxton T. Pope. Pp. 103-152, plates 21-37. March, 1918

4. Yana Terms of Relationship, by Edward Sapir. Pp. 153-173. : March, 1918

Vol. 14. 1. The Ianguage of the Salinan Indians, by J. Alden Mason. Pp. 1-154. Janitary, 1918

2. Clans and Moieties in Southern California, by Edward Winslow Gifford. Pp. 155-219, 1 figure in text. March, 1918

3. Ethnogeography and Archaeology of the Wiyot Territory, by Llewellyn L. Loud. Pp. 221-436, plates 1-21, 15 text-figures. December, 1918

4. The Wintin Hesi Ceremony, by S. A. Barrett. Pp. 437-488, plates 22-23, 3 figures in text. March, 1919

5. The Genetic Relationship of the North American Indian Languages, by Paul Radin. Pp. 489-502. May, 1919

Vol. 15. 1. Ifugao Law, by R. F. Barton. Pp. 1-186, plates 1-33. February, 1919 ........

2. Nabaloi Songs, by C. R. Moss and A. I. Kroeber. Pp. 187-206. May, 1919

Vol. 16. 1. Myths of the Southern Sierra Miwok, by S. A. Barrett. Pp. 1-28. March, $1919^{\circ}$

2. The Matrilineal Complex, by Robert H. Iowie. Pp. 29-45. March, 1919....

Volumes now completed:

Volume 1. 1903-1904. 378 pages and 30 plates

Volume 2. 1904-1907. 393 pages and 21 plates

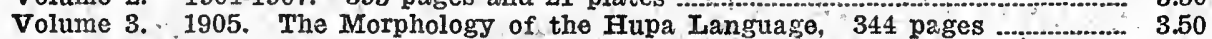

Volume 4. 1906-1907. 374 pages, with 5 tables, 10 plates, and map .................... 3.50

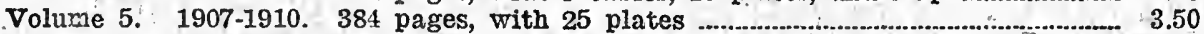

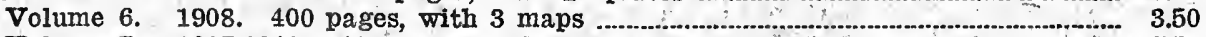

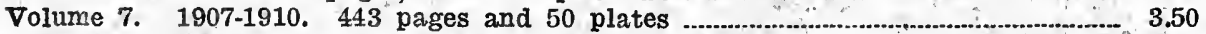

Volume 8. 1908-1910. 369 pages and 28 plates

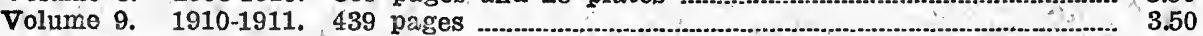

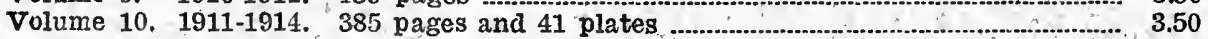

Volume 11. 1911-1916. 479 pages and 45 plates

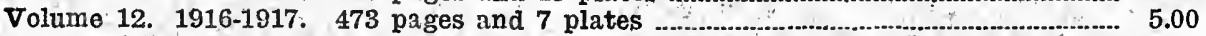

Note:-The University of California Publications are offered in exchange for the publications of learned societies and institutions, universities, and libraries. Complete lists of all the publications of the University will be sent upon request. For sample copies, lists of publications or other information, address the MANAGER OF THE UNIVERSITY PRESS, BERKELEY, CAIIFORNTA, U. S. A. All matter sent in exchange should be addressed to THE EXCHANGE DEPARTMENT, UNIVERSITY IIBRARY, BERKEIEY, CAIIFORNIA, U. S. A. 





